\title{
ISOMORPHISMS OF PICKERT-MOULTON PLANES
}

\author{
WILLIAM A. PIERCE
}

Pickert-Moulton Planes were defined more than a decade ago $[2$, p. 93], but the condition that two such planes be isomorphic has not appeared in the literature. Here, I shall give a simple criterion for the existence of such an isomorphism, and determine explicitly the most general isomorphism.

A Pickert-Moulton Plane over an ordered field $F$ has points $(x, y)$, and lines $[x=c],[y=c+r \circ x]$; where

$$
\begin{aligned}
r \circ x & =\operatorname{prx} & & \text { for } r, x<0, \\
& =r x & & \text { otherwise; }
\end{aligned}
$$

$p>0, c, r, x, y$ being in $F$. (In F. R. Moulton's classic model [1], the constant $p$ is 2 and $F$ is the field of real numbers.)

The (generalized) Moulton Planes which I defined in [3], have

$$
\begin{aligned}
r \circ x & =r x & & \text { for all } x \geqq 0 \\
& =\phi(r) \cdot x & & \text { for all } x<0
\end{aligned} \text { and all } r(\text { in } F) .
$$

Over an ordered field $F, \phi$ is an increasing function of $F$ on to $F$ (normalized by $\phi(0)=0, \phi(1)=1)$, restricted only by the condition: $x \rightarrow \phi(x)+x$ maps $F$ onto $F[3$, p. 429, Theorem 1]. The PickertMoulton Plane is a special "Moulton Plane" with $F$ ordered, and

$$
\begin{aligned}
\phi(r) & =r & & \text { for } r \geqq 0, \\
& =p r & & \text { for } r<0 .
\end{aligned}
$$

Thus the collineation theorems of [4] and [5] apply, in particular, to Pickert-Moulton Planes.

J. C. D. Spencer (Mrs. Yaqub) [6] has given explicit formulae for certain collineations on a Pickert-Moulton Plane. In [4] and [5], I determined the full collineation group for such a plane-in fact, for any Moulton Plane. Also, I characterized a Pickert-Moulton Plane among all Moulton Planes in terms of its collineation group; viz., as one supporting a collineation (onto itself) that displaces the ideal point on $[x=0]$. (Cf. [5, p. 650, Theorem 3].)

Theorem 1. Let $M_{\phi}(F)$ and $M_{\psi}(K)$ be Pickert-Moulton Planes over the respective ordered fields $F$ and $K$, where

Received by the editors February 15, 1966. 


$$
\begin{aligned}
\phi(r) & =r & \text { for } r(\text { in } F) \geqq 0, & \psi(r) & =r & \text { for } r(\text { in } K) \geqq 0, \\
& =\operatorname{pr} \text { for } r(\text { in } F)<0 ; & & =\text { qr } & \text { for } r(\text { in } K) & <0 .
\end{aligned}
$$

Then $M_{\phi}(F)$ and $M_{\psi}(K)$ are isomorphic if and only if there exists an order-preserving isomorphism $\alpha$ of $F$ onto $K$ such that $q=\alpha(p)$ or $q \cdot \alpha(p)=1$.

Proof. The general form of the collineation in [5, p. 650, Theorem 3] shows that $M_{\phi}(F)$ is isomorphic to $M_{\psi}(K)$ if and only if there is an affine collineation of $M_{\phi}(F)$ onto $M_{\psi}(K)$. This, by $[4$, p. 54, Theorem 1], is equivalent to the existence of an order-preserving isomorphism $\alpha$, from $F$ onto $K$, and constants $e, a \neq 0$ in $K$, such that either

$$
\begin{aligned}
\text { (Case (i)) } \quad \alpha \phi(r) & =\frac{\psi[a \cdot \alpha(r)+e]-\psi(e)}{\psi(a+e)-\psi(e)}, \text { or } \\
\text { (Case (ii)) } \quad \alpha(r) & =\frac{\psi[a \cdot \alpha \phi(r)+e]-\psi(e)}{\psi(a+e)-\psi(e)}
\end{aligned}
$$

for all $r$ in $F$.

Formulae (1) can be combined by putting

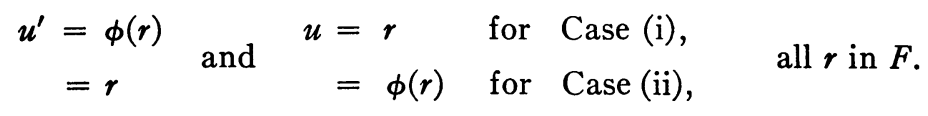

The composite formula is

$$
\alpha\left(u^{\prime}\right)=\frac{\psi[a \cdot \alpha(u)+e]-\psi(e)}{\psi(a+e)-\psi(e)},
$$

where

$$
u^{\prime}=u, \quad \text { for all } \quad u \geqq 0,=k u \quad \text { for all } \quad u<0 ;
$$

$k$ being $p$ in Case (i), but $p^{-1}$ in Case (ii). By definition,

$$
\begin{aligned}
\psi[a \cdot \alpha(u)+e] & =a \cdot \alpha(u)+e \quad \text { for } a \cdot \alpha(u)+e \geqq 0, \\
& =q a \cdot \alpha(u)+q e \quad \text { for } a \cdot \alpha(u)+e \leqq 0,
\end{aligned} \text { all } u \text { in } F .
$$

Substituting this in (3) gives

$$
\begin{aligned}
u^{\prime} & =\frac{\alpha^{-1}(a) \cdot u+\alpha^{-1}[e-\psi(e)]}{\alpha^{-1}[\psi(a+e)-\psi(e)]} \text { as }\left\{\left[\alpha^{-1}(a)\right] \cdot u+\alpha^{-1}(e)\right\} \geqq 0, \\
& =\frac{\alpha^{-1}(q a) \cdot u+\alpha^{-1}[q e-\psi(e)]}{\alpha^{-1}[\psi(a+e)-\psi(e)]} \text { as }\left\{\left[\alpha^{-1}(a)\right] \cdot u+\alpha^{-1}(e)\right\} \leqq 0,
\end{aligned}
$$


for all $u$ in $F$. Comparison of (4) and (5) shows that $e=\psi(e)=0$, and that the (unordered) pair

$$
\begin{aligned}
\left\{\alpha^{-1}[a / \psi(a)], \alpha^{-1}[q a / \psi(a)]\right\} & =[1, k]=[1, p] \text { in Case (i), } \\
& =\left[1, p^{-1}\right] \text { in Case (ii). }
\end{aligned}
$$

Thus, either $a / \psi(a)=\alpha(1)=1$ and $q a / \psi(a)=\alpha(k)$; or $a / \psi(a)=\alpha(k)$ and $q a / \psi(a)=\alpha(1)=1$. In either case, $q=\alpha(p)$ or $q \cdot \alpha(p)=1$.

We conclude that the required (order-preserving isomorphism) $\alpha$ exists if and only if $q=\alpha(p)$ or $q \cdot \alpha(p)=1$, and the theorem follows.

Corollary. If $\alpha$ is the identity (as it must be when $F=K=$ the real field), the condition for isomor $p$ hism reduces to $q=p$ or $q=p^{-1}$.

The isomorphisms between any two Moulton Planes were completely determined in [4] and [5]. It should be of interest, however, to exhibit the formulae for the special case of the Pickert-Moulton Planes; and this is done in Theorem 2 below. The notation of Theorem 1 will be retained.

THEOREM 2. (A) The most general affine isomorphism $\beta$ of $M_{\phi}(F)$ onto $M_{\psi}(K)$ sends $(x, y)$ to $\left(x^{\prime}, y^{\prime}\right)$; where $y^{\prime}=g \cdot \alpha(y)+d$ for all $y$ in $F$, and

$$
\begin{aligned}
x^{\prime}=\mu(x) & =g / a \cdot \alpha(x) & & \text { for } x^{\prime} \geqq 0, \\
& =g / \psi(a) \cdot \alpha(x) & & \text { for } x^{\prime}<0,
\end{aligned}
$$

all $x$ and nonzero constants $g, a$ in $F$.

For any $r$ in $F, \beta\left[P_{\infty}(r)\right]=P_{\infty}\left(r^{\prime}\right)$, where

$$
\begin{aligned}
\alpha(r) & =r^{\prime} / a & & \text { in Case (i) } \mu(1)>0, \\
& =\psi\left(r^{\prime}\right) / \psi(a) & & \text { in Case (ii) } \mu(1)<0 ;
\end{aligned}
$$

$\beta\left(Y_{\infty}\right)=Y_{\infty}^{c}$.

(B) The most general nonaffine isomorphism $\gamma$ of $M_{\phi}(F)$ onto $M_{\psi}(K)$ is $\sigma \beta$; with $\beta$ the general affine isomorphism (Part A), and $\sigma$ a special collineation on $M_{\phi}(F)$. Let $k$ be an arbitrary constant in $F$. For all $x$ and all $y \neq(-1-k)$ in $F, \sigma[(x, y)]=\left(x^{\prime}, y^{\prime}\right)$ where

$$
\begin{aligned}
& y^{\prime}=\frac{y+k}{y+k+1}, \\
x^{\prime}=\frac{x}{p \cdot(y+k+1)} & \text { for } x \geqq 0 \quad \text { and } y+1+k<0, \\
=\frac{x}{y+k+1} & \text { otherwise. }
\end{aligned}
$$


The map $\sigma$ is completed as follows: $\sigma[(x,-1-k)]=P_{\infty}(-1 / x)$ for all $x \neq 0 ; \sigma[(0,-1-k)]=Y_{\infty} ; \sigma\left(Y_{\infty}\right)=(0,1) ; \sigma\left[P_{\infty}(0)\right]=P_{\infty}(0) ;$ and

$$
\begin{aligned}
\sigma\left[P_{\infty}(r)\right] & =(1 / r, 1) & & \text { for } r>0, \\
& =(1 / p r, 1) & & \text { for } r<0,
\end{aligned}
$$

(C) The general nonaffine collineation $\gamma$ may be summarized explicitly as follows. If $y \neq-1-k$, and $x$ is in $F$, then $\gamma[(x, y)]=\left(x^{\prime}, y^{\prime}\right)$; where

$$
y^{\prime}=g \cdot \alpha[(y+k) /(y+k+1)]+d, \text { for all } y \text { in } F ;
$$

the line $y=-1-k$ in $M_{\phi}(F)$ maps onto the ideal line of $M_{\psi}(K)$; and the ideal line of $M_{\phi}(F)$ maps onto $\left\{y^{\prime}=g+d\right\}$ in $M_{\psi}(K) ; \gamma\left[P_{\infty}(0)\right]$ $=P_{\infty}(0) ; \gamma\left(Y_{\infty}\right)=(0, g+d) ;$ and $\gamma[(0,-1-k)]=Y_{\infty}$.

Case (i). $\mu(1)=g / a>0$.

For $x \neq 0, \gamma[(x,-1-k)]=P_{\infty}[a \cdot \alpha(-1 / x)]$;

$$
\begin{aligned}
\gamma\left[P_{\infty}(r)\right] & =(g /[a \cdot \alpha(r)], g+d) & & \text { for } r>0, \\
& =(g /[\psi(a) \cdot \alpha(p r)], g+d) & & \text { for } r<0 ;
\end{aligned}
$$

and

$$
\begin{aligned}
x^{\prime} & =[g / \psi(a)] \cdot \alpha\{x /[p \cdot(y+k+1)]\} & & \text { for } x \geqq 0 \text { and }(y+k+1)<0, \\
& =(g / a) \cdot \alpha[x /(y+k+1)] & & \text { for } x \cdot(y+k+1)>0, \\
& =[g / \psi(a)] \cdot \alpha[x /(y+k+1)] & & \text { for } x \leqq 0 \text { and }(y+k+1)>0 .
\end{aligned}
$$

Case (ii). $\mu(1)=g / \psi(a)<0$.

For $x \neq 0, \gamma[(x,-1-k)]=P_{\infty}\left\{\psi^{-1}[\psi(a) \cdot \alpha(-1 / x)]\right\}$;

$$
\begin{aligned}
\gamma\left[P_{\infty}(r)\right] & =(g /[\psi(a) \cdot \alpha(r)], g+d) & & \text { for } r>0, \\
& =(g /[a \cdot \alpha(p r)], g+d) & & \text { for } r<0 ;
\end{aligned}
$$

and

$$
\begin{aligned}
x^{\prime} & =(g / a) \cdot \alpha\{x /[p \cdot(y+k+1)]\} & & \text { for } x \geqq 0 \text { and }(y+k+1)<0, \\
& =[g / \psi(a)] \cdot \alpha[x /(y+k+1)] & & \text { for } x \cdot(y+k+1)>0, \\
& =(g / a) \cdot \alpha[x /(y+k+1)] & & \text { for } x \leqq 0 \text { and }(y+k+1)>0 .
\end{aligned}
$$

Proof. (A) The general affine isomorphism $\beta$ is given by $[4$, p. 54, Theorem 1], with $\psi(e)=e=0$ as in Theorem 1 above.

(B) The general nonaffine isomorphism $\gamma$, according to $[5$, p. 650 , Theorem $\left.3_{\mathrm{a}}\right]$, is $\tau \delta \beta$; where $\tau$ is a translation $(x, y) \rightarrow(x, y+k), \delta$ [called " $\alpha$ " in Theorem 3] is a special collineation of $M_{\phi}(F)$, and $\beta$ is the general affine collineation (Part (A) above).

The collineation $\tau \delta=\sigma$ (which reduces to $\delta$ in the special case 
$k=0$ ) can be obtained from [ 5, p. 655 , Corollary 3$]$ by putting $a=h$ $=0$ and $p=1$, replacing $y$ by $y+k$, and finally changing $q$ to $p$.

(C) The explicit $\gamma$ is obtained by calculating the resultant of $\sigma$ followed by $\beta$.

The proof is now complete.

\section{REFERENCES}

1. F. R. Moulton, A simple non-Desarguesian plane, Trans. Amer. Math. Soc. 3 (1902), 192-195.

2. G. Pickert, Projektive Ebenen, Springer-Verlag, Berlin, 1955.

3. W. A. Pierce, Moulton planes, Canad. J. Math. 13 (1961), 427-436. 62.

4. - Collineations of affine Moulton planes, Canad. J. Math. 16 (1964), 46-

5. - Collineations of projective Moulton planes, Canad. J. Math. 16 (1964), $637-656$.

6. J. C. D. Spencer, On the Lenz-Barlotti classification of projective planes, Quart. J. Math. Oxford Ser. (2) 2 (1960), 241-257.

LyNDONVILLE, VERMONT 